Editorial

\title{
Schizophrenia: yesterday's concept
}

\author{
I Brockington
}

Queen Elizabeth Psychiatric Hospital, Mindelsohn Way, Birmingham, BI5 2Q, UK

\section{Introduction}

The classification of the psychoses is one of psychiatry's main challenges. The distinction between manic depressive insanity and dementia praecox (affective psychosis and schizophrenia), had, when introduced by Kraepelin, a convenient simplicity, and provided psychiatry with an orientation to guide clinical work and focus research. For 100 years, the 'two entities principle' has been our nosological keystone, and the concept of schizophrenia has taken a grip on the minds of the profession and the public - an idea which symbolizes psychiatry, powerful in the clinics, the law courts and research councils. However, although this word is in the mind and on the tongue of most psychiatrists most days of their professional lives, some have felt uneasy, and have doubted whether the notion had clarity enough to convey anything precise and unambiguous about our patients. What is 'schizophrenia'?

In the broad terms used in the glossary of the 8th Revision of the International Classification of Diseases, Injuries and Causes of Death (1968), schizophrenia is 'A fundamental disturbance of the personality involving its most basic functions, which give the normal person his feeling of individuality, uniqueness and self-direction'.

To reach a consensus on a more precise definition, however, has proved difficult. These difficulties came out into the open in the 1960s, when the first empirical studies on the reliability of psychiatric diagnosis were published (Kreitman, 1961), and the US/UK Diagnostic Project exposed the differences between the American and British use of the term (Cooper et al, 1972). Hempel (1961) urged psychiatrists to use operational definitions. They responded enthusiastically, and a tower of
Babel of precise but conflicting criteria was soon erected. These have not solved the problem of definitional disagreement, but they have made it easier to measure.

The technique used to study the relationship between definitions is the 'polydiagnostic technique' introduced by Hawk et al (1975), and independently by Kendell (Brockington et al, 1978), and thoroughly exploited by Berner (Berner et al, 1982), in which all the definitions are applied to the same series of patients. This technique was applied to the British series of 250 consecutive admissions collected for the US/UK Diagnostic Project ('the Netherne series'). Eighty-five patients were given a hospital diagnosis of schizophrenia, compared with 163 in the corresponding American series - the Brooklyn series - indicating that American psychiatrists in the 1960s used 'schizophrenia' almost coterminously with 'psychosis'. The project team of co-trained experts (Cooper, Kendell and Sartorius), using the International Classification of Diseases, made the diagnosis in 65 patients. The Catego computer program, based largely on Schneider's 'first rank symptoms', placed only 55 patients within the classes which corresponded to schizophrenia (mainly 'S +'). When the Research Diagnostic Criteria were applied, which required similar 'characteristic' symptoms but excluded patients with a manic or depressive syndrome, only 28 patients qualified. When DSM III was applied, requiring additional evidence of chronicity and social decline, only 19 qualified. Assuming that the Brooklyn series is equivalent to the Netherne series, the number of patients given the diagnosis of schizophrenia by American psychiatrists melted away from 163 to 19 in 15 years, but the same word was used throughout.

There must be something profoundly wrong with a concept which has proved so unstable in its usage, 
geographically and historically. Its interpretation is strongly influenced by local tradition, and is subject to social rather than scientific constraints. An orthodoxy of diagnostic rules can be decreed by a professional hierarchy, but only within its own parish. During the last decade, we have witnessed a vigorous attempt by the American Psychiatric Association to persuade the psychiatric community to accept an arbitrary definition (which changes every few years), in order to ensure the equivalence of patient groups studied in different institutions. This is admirable, provided that it does not suppress nosological innovation, or guillotine discussion of the fundamental flaws in the concept of schizophrenia.

\section{A nosological fiction}

Why have psychiatrists failed to reach agreement about the diagnosis of schizophrenia? In a nutshell, it is because schizophrenia is an idea without a single defining principle.

The nosologist's role is to develop a system of ideas and a nomenclature for clinical and scientific use. These ideas are the more powerful the more unequivocally each reflects a single aspect of disease. The process of developing a disease concept begins with a key observation about some abnormality shared by a group of patients. This is the essence (intension) of the disease. After this has been recognized, the hunt is on to explore the boundaries (extension) of the clinical phenomenon - what else is to be included, and what is to be excluded. The problem with schizophrenia is that its intention is ambiguous, and therefore the attempt to reach a consensus on its extension is doomed to frustration. The clarity of Kraepelin's original idea has been lost, and it now has, not one, but two or even three totally different defining principles.

The germ of Kraepelin's idea of 'dementia praecox' was convergence to a defect state, ie this disease was defined by a final common path, as in Bright's disease or cor pulmonale. As he wrote, 'I got the starting point of the line of thought... from the overwhelming impression of states of dementia quite similar to each other which developed from the most varied initial clinical symptoms.' Starting with hebephrenia, catatonia or paranoid hallucinatory states, the patients followed a pernicious course ending in 'A peculiar destruction of the internal connections of the psyche, with the most marked damage to the emotional life and volition' (Kraepelin, 1919).

In a theoretical formulation of the dysfunction of patients who followed this common path, a se- cond defining principle was soon introduced, that of 'psychic fission'. This idea is derived from the group of symptoms, present in about half the patients, which includes verbal hallucinosis, echo phenomena and other automatisms, sometimes associated with explanatory delusions (eg possession or control). These symptoms all imply a dualism or schism within the individual, in which the will is eroded by competing thoughts or impulses emanating from a rival focus. Symptoms of this kind ('the nuclear syndrome') have been widely accepted as pathognomonic of the disease, and the 'first rank symptoms of Schneider' are largely in this domain. They are emphasized in the ICD8 glossary quoted above. This defining principle is, however, different from Kraepelin's original idea, and these symptoms are not significantly correlated with prognosis (Brockington et al, 1978).

Neither the defect state nor the nuclear syndrome, however, exhaust the psychopathology of schizophrenia, since only a minority of patients show them in florid form. A large and, some would think, the most important group of symptoms cannot be related to them - that concerned with 'delusional systems' and associated phenomena (bizarre behaviour, non-verbal hallucinosis and idiosyncratic communication), which stem from the schizophrene's withdrawal from consensual contact - in a sense, his 'autism'. Delusions do not have the claim to specificity which defect or schism have, since they are ubiquitous in psychosis. It would be hard to pin-point a variety of delusions, in form, content or delusional mechanism, which is characteristic of schizophrenia; nevertheless they feature prominently in the definitions. This is, therefore, a third, radically different, defining principle of schizophrenia. These three core concepts cannot be derived from each other. Thus, the richness of the psychopathology of schizophrenia, which Kraepelin and Bleuler described so vividly, is its greatest weakness.

The definition of a disease is facilitated if patients, who belong to a nosological taxon, stand out distinctly from all other patients. This isolation of a patient group requires that, in terms of some variable, there is a scarcity of intermediate forms. The statistical technique of discriminant function analysis is an appropriate method to demonstrate such areas of rarity. Over the years it has been applied a number of times (Kendell and Gourlay, 1970; Brockington et al, 1979; Cloninger et al, 1985), and has consistently failed to demonstrate a boundary between schizophrenia and the affective psychoses. The most recent attempt, which involved a 4-group canonical variate analysis, showed 
repeatedly that manic depressive (bipolar) disorders were distinct, but patients with schizophrenia (defined by DSM III) belonged to a clinical continuum in which schizophrenia gradually merged with schizoaffective depression and depression (Brockington et al, 1991). The reason for this failure was evident. The psychopathology of schizophrenia can be analysed into several different factors - verbal hallucinosis and passivity phenomena, delusion formation, defect symptoms and social deterioration (corresponding to the defining principles mentioned above), which have different frequencies and are, for the most part, continuously distributed within a population of psychotic patients. It is inevitable that patients with schizophrenia lie on a continuum, with an abundance of interforms. Schizophrenia is a conceptual artefact which does not correspond to any natural grouping of patients.

\section{The penalties of a weak diagnostic concept}

Psychiatry pays a high price for its allegiance to the weak concept of schizophrenia. Its incoherence, and the vacillation over its meaning have damaged the reputation of the speciality. This is an idea which can hardly be defended in discussion with physicians or even intelligent laymen.

The two entities principle reduced the complexity of clinical assessment, and facilitated the investigation of mental illness. In terms of this dichotomy, the clinical study of a psychotic patient has become rather simple. Armed with structured interviews and an operational definition, a neuroscientist can soon assign a patient, suffering from a complex derangement over many years and through multiple episodes, to its kraepelinian category, which can then be related to some biological variable. It takes less time to determine the psychiatric state for research purposes than it takes to assess the haemodynamic state for clinical purposes. Given the complexity of the organ which is the seat of psychiatric illness, the clinical study of its failure must be among the most complex exercises of medicine, and we oversimplify at our peril.

The results of four generations of research into the causes of schizophrenia have been meagre. There is a genetic element, which to some extent breeds true. Dopamine has a role (not confined to schizophrenia) and there is some form of brain damage, perhaps in the temporal lobes. A hundred years of research has not yielded a single pathognomonic neuroscientific finding or specific therapy, and this 'disease entity' is still without external validation. For this fiasco, nosologists must take their share of the blame. Progress has been ham- pered by the heterogeneous groups which scientists have been required to study, under this portmanteau term. It is unreasonable to expect that patients who are heterogeneous not only in their symptoms, but also in their course and outcome, share a common cause.

There is competition between diagnostic concepts, and the obsession with schizophrenia has been pursued at the expense of other more promising lines of enquiry. Smaller, more homogeneous entities have been sucked in by the gravity of the big idea, and annihilated. Research has been concentrated on a core of patients with the most complex psychopathology and a chronic course. Reciprocally, the monosymptomatic hallucinatory, delusional and defect states, hebephrenia and the psychoses defined by an aetiological association (such as puerperal and menstrual psychoses), have been neglected, and starved of attention and funding.

The greatest damage, however, has been in the stifling of clinical curiosity. Psychiatry is a clinical science whose patent is to study disease, and whose privilege is to have unlimited access to patients. The task of 'observing spontaneous phenomena' urged on us by Sir Thomas Lewis (1930), is in its early stages. Because they can put such a name to the totality of a patient's experience and behaviour, many psychiatrists have lost their inquisitiveness about the inscrutable mysteries of psychotic phenomena. Schizophrenia does not help us to understand what is wrong with our patients. It has given many psychiatrists an unjustifiable complacency, an illusion of understanding, a false authority.

\section{Replacing schizophrenia}

Any challenge to a concept in daily and universal use is bound to provoke the demand for a more satisfactory replacement. This is justified, although one should not underestimate the value of acknowledging the bankruptcy of an idea, since this is a potent stimulus to innovation. The notion of schizophrenia has monopolized the thinking of psychiatrists so completely and for so long, that it is difficult to imagine a situation in which we are "living without schizophrenia' (Boyle, 1990). Nevertheless, we have already moved a long way towards this position. American psychiatrists use the diagnosis for about one sixth of the patients who would have received it 20 years ago, and British psychiatrists, who still seem to rely largely on Schneider's opinions, for about one half. Schizoaffective mania, paranoid disorder, delusional depression and cy- 
cloid psychosis have already replaced schizophrenia over much of its former spectrum. The DSM III definition has restricted the term to patients with a full deployment of symptoms and defects. Inevitably, this trend will continue, and the category will be further eroded as new distinctions are recognized, and will wither away.

A diagnostic category has many functions. In clinical practice, it acts as a shorthand to condense information, facilitate communication with other practitioners and open up lines of investigation and intervention. In the Courts it confers a medical explanation of deviant behaviour, and socially it supports the sick role. In certain types of research (especially genetics and epidemiology), there is a need for dichotomous groupings. Although categorical diagnosis is an oversimplification inappropriate for most clinical and scientific purposes, we cannot dispense with it. Without schizophrenia it will be difficult to classify the rump of chronic disorders with psychopathology in several areas. This will be the ditch which the diehards will defend. However, the classification of délires chroniques in terms of delusional mechanism, worked out by French psychiatrists, goes some way to solve the problem (Pichot, 1982).

For most clinical work, and for many areas of research, categorical diagnosis is unnecessary and harmful. When dealing with patients with intractable polysymptomatic illnesses - with perceptual and thinking disorders, defects and handicaps (both primary and secondary), often associated with affective and neurotic symptoms - the psychiatrist's role is to analyse the clinical record, identify and evaluate each component of the patient's mental life, and devise a range of interventions, dealing with each on its merits. A medical approach based on categorical diagnosis tends to divert attention from this task, to the detriment of patient care.

This is also true of research. Much research works just as well with an analytic approach. Neurochemical and neurophysiological studies, for example, can employ more powerful statistical techniques, when using parametric clinical measures, applied to 'dimensions' of psychopathology.

The idea that schizophrenia is a 'disease entity' misleadingly holds before us the mirage of spectacular discoveries like those made in infectious or nutritional disease. These are not appropriate paradigms. A better analogy would be that of organ failure, or (in view of the complexity of the central nervous system) of the dysfunction of certain systems within the brain. The assumption of a unitary disease encourages a reductionism, which skips the laborious task of unravelling the pathogenesis of clinical ef- fects, and goes straight for prime causes. An important thrust in medical research, brilliantly successful in internal medicine, has been the exegesis of symptoms in terms of disturbed physiology. In psychiatry, the corresponding task is to elucidate the impact of events (whether they be social or biological) on the normal 'structure' (biochemical, physiological or psychological) of the mind and brain. Schizophrenia is 'a multiconditioned lifeprocess, occurring in people with a particular vulnerability, interacting with complex life-events and circumstances' (Ciompi, 1984). Beginning with the acknowledgement that schizophrenia is neither an entity nor a unity, we should analyse its psychopathology into its elements, and search for the factors which determine each of them.

Bentall et al (1988) urge that we concentrate our enquiries on individual symptoms. This is a valuable suggestion, but factor analysis has endorsed three groups of symptoms which correspond to the three historic themes mentioned above - schism, delusion formation and defect - and these seem to correspond to three systems of brain function.

The first, which is deranged in verbal hallucinosis and passivity phenomena, governs the integration of the person, and the control of the stream of thought. Disorders of this kind have a narrow range of aetiological associations, and may be based on a localised neuroanatomical lesion. They often occur as a monosymptomatic state, or associated with a delusional explanation.

The second system, which is implicated in delusions, is concerned with the development and maintenance of a schema relating the individual to his social milieu. Delusions are ubiquitous in mental illness, and are of many types as well as varied content, and in a discussion of schizophrenia it is necessary to focus on delusional systems which evolve progressively by reasoning and are maintained over long periods. Their evolution is driven by affects, and influenced by many factors including the loss of feedback in subjects isolated by language or cultural barriers, and the need to resolve conflicts and maintain self-esteem. Once established, such ideas are maintained by a different set of factors including the inertia that all ideas have, the difficulty of retracting views expressed in public and justifying actions which would otherwise be unpardonable, and by a number of vicious circles including social isolation, the restriction of the field of interest, hypervigilance and the search for confirmatory evidence biassing perception, the loss of social effectiveness, and behaviour which provokes a confirmatory response from others. Thus, the explication of delusional systems will involve many 
different factors, some biological, some psychological and some social. It is common to encounter patients in whom such systems are the predominant or only symptom present, and they are suitable for the investigation of delusional mechanisms.

The third system is concerned with motivation and social effectiveness, and is disordered in the defect state. It was Kraepelin who drew attention to the convergent path of deterioration in psychoses of diverse origin. This important observation poses the question, 'What are the factors which lead to deeper social incapacity?' We need to tease apart the causes of social decline, just as we need to understand the dynamics of the right heart in pulmonary disease, and the way in which renal destruction increases arterial resistance and depresses the bone marrow. Many would now accept that these factors are legion, and include social handicaps and vulnerabilities present before the first sign of psychosis (eg paranoid or schizoid attitudes, schizotypal traits) as well as a skein of social, biological and iatrogenic factors which supervene as complications of psychotic episodes.

\section{Conclusion}

It is important to loosen the grip which the concept of 'schizophrenia' has on the minds of psychiatrists. Schizophrenia is an idea whose very essence is equivocal, a nosological category without natural boundaries, a barren hypothesis. Such a blurred concept is 'not a valid object of scientific enquiry' (Bentall et al, 1988). It is a cloak for ignorance and exposes psychiatry to ridicule. As a model of psychosis it is an oversimplification, which serves the interests of neither scientists nor patients. It is time to abandon this concept, with its history of semantic wrangles. In a clinical discipline, nosology is paramount. Psychiatry needs to be released from the straitjacket of this idea into a new vigour and freedom of enquiry.

\section{References}

Bentall RP, Jackson HF, Pilgrim D (1988) Abandoning the concept of 'schizophrenia': some implications of validity arguments for psychological research into psychotic phenomena. Br J Clin Psychol 27, 303324

Berner P, Katschnig H, Lenz G (1982) Poly-diagnostic approach: a method to clarify incongruences among the classification of the functional psychoses. Psychiatric J Univ Ottawa 7, 244-248

Boyle M (1990) Schizophrenia: a Scientific Delusion, Routledge, London

Brockington IF, Kendell RE, Leff JP (1978) Definitions of schizophrenia: concordance and prediction of outcome. Psychol Med 8, 387-398

Brockington IF, Kendall RE, Wainwright S, Hillier VF, Walter J (1979) The distinction between the affective psychoses and schizophrenia. $B r J$ Psychiatry 135, $243-248$

Brockington IF, Roper A, Buckley M, Copas J, Andrade C, Wigg P, Farmer A, Kaufman C, Hawley R, Meltzer HY (1991) Bipolar disorder, cycloid psychosis and schizophrenia: a study using 'lifetime' psychopathology ratings, factor analysis and canonical variate analysis. Eur Psychiatry 6, 223-236

Ciompi L (1984) Is there really a schizophrenia? The longterm course of psychotic phenomena. BrJ Psychiatry $145,636-640$

Cloninger CR, Martin RL, Guze SB, Clayton PJ (1985) Diagnosis and prognosis in schizophrenia. Arch Gen Psychiatry 42, 15-25

Cooper JE, Kendell RE, Gurland BJ, Sharpe L, Copeland JRM, Simon R (1972) Psychiatric Diagnosis in New York and London. Oxford University Press, London

Hempel CG (1961) Introduction to problems of taxonomy. In: Field Studies in the Mental Disorders (Zubin J, ed) Grune \& Stratton, New York, 3-22

Hawk AB, Carpenter WT, Strauss JS (1975) Diagnostic criteria and 5-year outcome in schizophrenia. Arch Gen Psychiatry 32, 343-347

Kendell RE, Gourlay J (1970) The clinical distinction between the affective psychoses and schizophrenia. $B r J$ Psychiatry 117, 261-266

Kraepelin E (1919) Dementia Praecox, translated by RM Barclay, Livingston, Edinburgh

Kreitman N (1961) The reliability of psychiatric diagnosis. J Ment Sci $107,876-886$

Lewis T (1930) Observations on research in medicine: its position and its needs. $\mathrm{Br}$ Med J 1, 479-483

Pichot P (1982) The diagnosis and classification of mental disorders in French-speaking countries: background, current views and comparison with other nomenclatures. Psychol Med 12, 475-492

World Health Organization (1968) A Glossary of Mental Disorders. London, HASD 